Check for updates

Cite this: RSC Adv., 2019, 9, 38055

Received 18th August 2019

Accepted 11th November 2019

DOI: $10.1039 / c 9 r a 06466 d$

rsc.li/rsc-advances

\title{
Resonant waveguide grating based assays for colloidal aggregate detection and promiscuity characterization in natural products $\dagger$
}

\begin{abstract}
Rong Wang, ${ }^{\text {ab }}$ Jixia Wang, ${ }^{\text {ad }}$ Yanfang Liu, ${ }^{\text {*ad }}$ Xiuli Zhang ${ }^{\star c}$ and Xinmiao Liang (D) ad
Small molecules, including natural compounds, in aqueous buffer that self-associate into colloidal aggregates is the main cause of false results in the early stage of drug discovery. Here we reported resonant waveguide grating (RWG) based assays to identify natural compound aggregation and characterize its influence on membrane receptors in living cells. We first applied a cell-free aggregation assay to determine compound critical aggregation concentration (CAC) values. Then we characterized the aggregators' influence on membrane receptors using three types of dynamic mass redistribution (DMR) assays. Results showed that colloidal aggregates may cause false activity in DMR desensitization assays; some of the false activities can be implied by the large response in DMR agonism assays and can further be identified by DMR antagonism assays. Furthermore, the aggregation mechanism was confirmed by addition of $0.025 \%$ tween-80, with cell signals attenuated and potency decreased. Finally, these observations were used for aggregate examination and promiscuity investigation of a traditional herbal medicine, Rhodiola rosea, which ultimately led to the revealing of the true target and reduced the risk of a bioactivity tracking process at the very first stage. This study highlights that the RWG based assays can be used as practical tools to distinguish between real and false hits to provide reliable results in the early stage of drug discovery.
\end{abstract}

\section{Introduction}

High-throughput screening (HTS) technologies are vital tools for discovering hit compounds in the pharmaceutical industry. ${ }^{1}$ However, the HTS programs are often challenged by the frequent occurrence of false activities when special care is not taken. ${ }^{2}$ The origins of false-active results include chemical reactivity, ${ }^{3}$ covalent binding, ${ }^{4}$ and interference in assay readout like absorbance, ${ }^{5}$ fluorescence, ${ }^{6}$ or luminescence. ${ }^{7}$ The dominant mechanism of the false activities, however, is the formation of colloidal aggregates in the aqueous assay buffer. ${ }^{8,9}$ It is reported that about $19 \%$ of compounds from a random selected library can form aggregators at the screen concentrations typically used in HTS. ${ }^{10}$ Once formed, these aggregators can cause artificial inhibition of soluble enzymes ${ }^{\mathbf{1 0 , 1 1}}$ and membranebounded receptors ${ }^{\mathbf{1 2}}$ through mechanisms like partial

${ }^{a}$ Key Lab of Separation Science for Analytical Chemistry, Dalian Institute of Chemical Physics, Chinese Academy of Sciences, Dalian 116023, China.E-mail: liuyanfang@ dicp.ac.cn

${ }^{b}$ University of Chinese Academy of Sciences, Beijing 100049, China

${ }^{c}$ College of Pharmaceutical Science, Soochow University, Suzhou 215123, China. E-mail: zhangxl@suda.edu.cn

${ }^{d}$ DICP-CMC Innovation Institute of Medicine, Taizhou 225300, China

$\dagger$ Electronic supplementary information (ESI) available. See DOI: 10.1039/c9ra06466d unfolding, dynamics restraining or protein sequestration, ${ }^{\mathbf{1 1 , 1 3 , 1 4}}$ they can also affect cell growth by reducing compound penetration into cells. ${ }^{15}$

Natural products (NPs), an important source of pharmaceutically interesting compounds, also suffered pains from aggregation phenomenon. Some compounds that are widely present in natural products have already been flagged as aggregators including flavanol, polyphenol, and other highly lipophilic or conjugated compounds. ${ }^{\mathbf{1 6 , 1 7}}$ In the classical NP program for tracking active compounds, samples are often initially tested as multicomponent mixtures. ${ }^{18}$ Consequently, the false active compounds not only act as problematic hits in themselves, but also play as metabolic matrix, interfering with the screening results, wasting the separation efforts and masking the activities of real hits. ${ }^{19-23}$ Therefore, NPs need careful scrutiny for the elimination of potential false hits at the very beginning of the program.

Several methods can be used for the direct detection of compound aggregation, such as transmission electron microscopy (TEM), ${ }^{11,24}$ dynamic light scattering (DLS) ${ }^{\mathbf{1 0 , 2 5}}$ and nuclear magnetic resonance (NMR). ${ }^{26-29}$ However, these approaches are lack of throughput or simplicity, and they need additional apparatus to screen assays, which may not be available in common pharmacological laboratory. Recently, the optical resonant waveguide grating (RWG) biosensor incorporated into 384-well microplate has been utilized for aggregate detection, 
which exhibited simplicity and high-throughput. ${ }^{30,31}$ Apart from aggregate detection, the RWG biosensor was well-known for its use in biomolecular interaction and cellular analysis, ${ }^{32}$ especially for the measurement of ligand-induced dynamic mass redistribution (DMR) signals in cells. ${ }^{33,34}$ The intrinsic sensitivity of the RWG is very high, and is very suitable for living cell sensing. ${ }^{35,36}$ In addition, the DMR assay can provide noninvasive, holistic, pathway sensitive and multi-assay format measurement, ${ }^{37,38}$ therefore a more comprehensive observation of aggregators' behavior could be obtained.

Here we applied RWG based assays to first identify natural compound aggregation, and then characterized their influence on membrane receptors in living cells. We further asked if these observations could be used for screening real NP sample to avoid the useless efforts in NP-based research programs.

\section{Experimental}

\subsection{Materials}

Salvianolic acid C, proanthocyanidins, luteolin, physcion were purchased from Shanghai Yuanye Bio-technology Co., Ltd. (China). Quercetin, genistein, silybin and curcumin were purchased from Chengdu Push Bio-technology Co., Ltd. (China). All the above compounds were with the HPLC purities of more than 97\%. Tween-80 was purchased from Beijing LabLead Biotech Co., Ltd. (China). Acetylcholine chloride, hyoscyamine and zaprinast were obtained from Sigma Chemical Co. (St Louis, MO, USA). ML145 was obtained from Tocris Bioscience Co. (St. Louis, MO, USA).

Epic® 384-Well Cell Assay Microplate (Cat. no. 5040) was obtained from Corning Incorporated (Corning, NY, USA). Human colorectal adenocarcinoma HT-29 cell line was obtained from the Cell Bank of Shanghai Institute of Cell Biology, Chinese Academy of Sciences.

For aggregation assay, salvianolic acid C, proanthocyanidins, luteolin, physcion, quercetin, genistein, silybin, curcumin, Rhodiola rosea extract were stocked in at $100 \mathrm{mg} \mathrm{mL}^{-1}$. For cell assays, salvianolic acid $\mathrm{C}$, proanthocyanidins, quercetin and curcumin were stocked at $200 \mathrm{mM}$, Rhodiola rosea extract was stocked at $100 \mathrm{mg} \mathrm{mL} \mathrm{m}^{-1}$. Acetylcholine was stocked at $1 \mathrm{M}$, zaprinast was stocked at $5 \mathrm{mM}$, ML145 and hyoscyamine were stocked at $100 \mathrm{mM}$. All compounds were dissolved in 100\% dimethyl sulfoxide (DMSO).

The assay buffer was $1 \times$ Hank's balanced salt solution (HBSS), $20 \mathrm{mM}$ HEPES, pH 7.2. The detergent assay buffer was $1 \times$ Hank's balanced salt solution (HBSS), 20 mM HEPES, 0.1\% tween-80 (v/v), pH 7.2. All compounds were diluted with above buffers to assay concentrations.

\subsection{Cell culture}

HT-29 cells were cultured using McCoy's 5A Medium (\#DY0324S0414L, Sango Biotech, Shanghai, China) with 10\% fetal bovine serum (\#10099141, Gibco, Life Technologies), $50 \mu \mathrm{g}$ $\mathrm{mL}^{-1}$ penicillin and $100 \mu \mathrm{g} \mathrm{mL}^{-1}$ streptomycin in a humidified $37{ }^{\circ} \mathrm{C} / 5 \% \mathrm{CO}_{2}$ incubator.

\subsection{Cell-free aggregation assay}

The aggregation assay was performed using an Epic BT system (Corning, NY, USA). The Epic 384-Well Cell Assay Microplates were first soaked with $30 \mu \mathrm{L}$ assay buffer and equilibrated in the Epic reader for 1 hour. After a 2 min baseline measurement, 10 $\mu \mathrm{L}$ of compound dilution series in buffer were added to each well. A range of final DMSO concentrations was tested to establish the background response. The maximum final DMSO concentration was $1 \%$. After adding compounds, the microplate was returned immediately to the Epic reader and signals were recorded for 1 hour. Each of the assays was performed at least three times with excellent reproducibility.

\subsection{Dynamic mass redistribution assays}

All DMR assays were performed using an Epic BT system (Corning, NY, USA).

Cells were directly seeded in Epic 384-Well Cell Assay Microplates and cultured for $\sim 24 \mathrm{~h}$ to form a monolayer in the cell culture medium. After being washed, the cells were maintained with $30 \mu \mathrm{L}$ assay buffer for $1 \mathrm{~h}$ in the Epic system.

For DMR agonism assay, a 2 min baseline was first established, followed by adding samples and monitoring the sampleinduced DMR signals for $1 \mathrm{~h}$. Then, the sample treated cells were reused immediately for DMR desensitization assay. A 2 min baseline was first established, followed by adding receptor agonists and monitoring the DMR signals for another $1 \mathrm{~h}$. The receptor agonists for GPR35, M3, NTSR and EGFR were zaprinast $(5 \mu \mathrm{M})$, acetylcholine $(80 \mu \mathrm{M})$, neurotensin $(2 \mathrm{nM})$, and EGF (2 nM), respectively.

For DMR antagonism assay, HT-29 cells were first pretreated with receptor antagonists for $10 \mathrm{~min}$, then a $2 \mathrm{~min}$ baseline was established, followed by adding $100 \mu \mathrm{M}$ compounds and monitoring the DMR signals for $1 \mathrm{~h}$. The receptor antagonists for GPR35 and M3 were ML145 and hyoscyamine, respectively.

\subsection{Data analysis}

All DMR data were acquired using Epic Imager software (Corning, NY, USA) and processed with Imager Beta 3.7 (Corning), Microsoft Excel 2013 and GraphPad Prism 6.02 (GraphPad Software Inc., San Diego, CA, USA). All DMR signals were background corrected. All $\mathrm{EC}_{50}$ or $\mathrm{IC}_{50}$ values described were calculated based on the maximal amplitude of the DMR signals. All DMR data were from two independent measurements, each in at least duplicate $(n=4)$.

\subsection{Extraction and sample treatment of Rhodiola rosea}

The root of Rhodiola rosea was crushed and extracted with $70 \%$ ethanol under ultrasonic for $1 \mathrm{~h}$. Then, the solvent was removed by rotary evaporation to acquire the crude extract. The treated extract was obtained by processing the crude extract with a quaternary ammonium ion exchange resin. 


\subsection{HPLC analysis of the crude extract and the treated extract of Rhodiola rosea}

The chromatographic analysis was carried out on Alliance HPLC with UV detector (Waters, Milford, USA). The column used was Unitary C18 (4.6 × $250 \mathrm{~mm}, 7 \mu \mathrm{m}$, Acchrom, Beijing, China). The mobile phases were A: methanol; B: water (containing $0.1 \%$ formic acid, v/v). Mobile phase gradient was started at $5 \%$ A for $5 \mathrm{~min}$, and then shifted from 5-95\% A in 5-45 min, and finally kept at $95 \% \mathrm{~A}$ for another $10 \mathrm{~min}$. The flow rate was 0.7 $\mathrm{mL} \min ^{-1}$. Column temperature were $30{ }^{\circ} \mathrm{C}$. The UV detection wavelength was $270 \mathrm{~nm}$.

\section{Results and discussion}

\subsection{Principles of cell-based DMR assay and cell-free aggregation assay}

The major principle of RWG biosensor is to use evanescent wave to record the local refractive index change $(\Delta n)$ about $150 \mathrm{~nm}$ depth near the sensor surface ${ }^{39}$ and output the signal as wavelength shift of reflected resonance light. ${ }^{40}$ The biosensor response, in terms of wavelength shift $\Delta \lambda$, is a linear function of the change in refractive index $\Delta n:^{36}$

$$
\Delta \lambda=(-47 \pm 37) \mathrm{pm}+\Delta n(113850 \pm 1257) \mathrm{pm}
$$

The imager is very sensitive and was reported to has a limit of detection down to $10^{-4}$ RIU for refractive index change under practical conditions. ${ }^{36}$

For cell environment, the refractive index of a given volume is largely determined by the concentrations of biomolecules such as proteins. ${ }^{33}$ When activated by compounds, the cellular proteins within the sensing volume will go through relocalization, resulting in shifted reflected wavelength (Fig. 1a). Generally, most GPCR receptors on native cells can obtain 100-
$500 \mathrm{pm}$ saturated response, which corresponds to $1.3 \times 10^{-3}$ to $4.8 \times 10^{-3}$ RIU for refractive index change. The real-time signal induced by the cellular local density change is also termed as dynamic mass redistribution (DMR).

For cell-free environment, the refractive index is mainly affected by the chemical or physical change like the formation of aggregators. When compound with low concentrations were added into the buffer to form soluble monomers, neglectable responses would be observed. Whereas upon increasing the concentration, the compound assembled into aggregators and caused a significant change in the local refractive index, leading to shifted reflected wavelength (Fig. 1b). The higher the aggregator concentration, the higher the response (Fig. 2a). However, not all compounds tend to display wavelength shift. For nonaggregate compounds that dissolve in monomer state across all the screening concentrations in the buffer, no wavelength shift would be observed for all the concentrations (Fig. 2b). Since the aggregation assay is a real-time measurement, the response curve indicates the local environment dynamics. The slightly fluctuation at $2 \mathrm{~min}$ indicated the solution disturbance right after adding sample (Fig. 2a and b), and the slow increase during 2-15 min indicated aggregator increasement near the bottom (Fig. 2a).

\subsection{Cell-free aggregation assay for CAC determination}

We first performed cell-free aggregation assay in 384-microplate that incorporated with RWG biosensors to investigated the critical aggregation concentrations (CACs) of eight natural compounds, including curcumin, genistein, luteolin, silybin, quercetin, procyanidins, salvianolic acid $\mathrm{C}$ and physcion. These compounds came from multiple high-throughput screening projects and the analogues of frequent aggregators. ${ }^{16,41,42}$ For each compound, a range of concentrations was tested. To determine the CAC values, the responses of different concentrations at fixed time were collected and analyzed, the responses
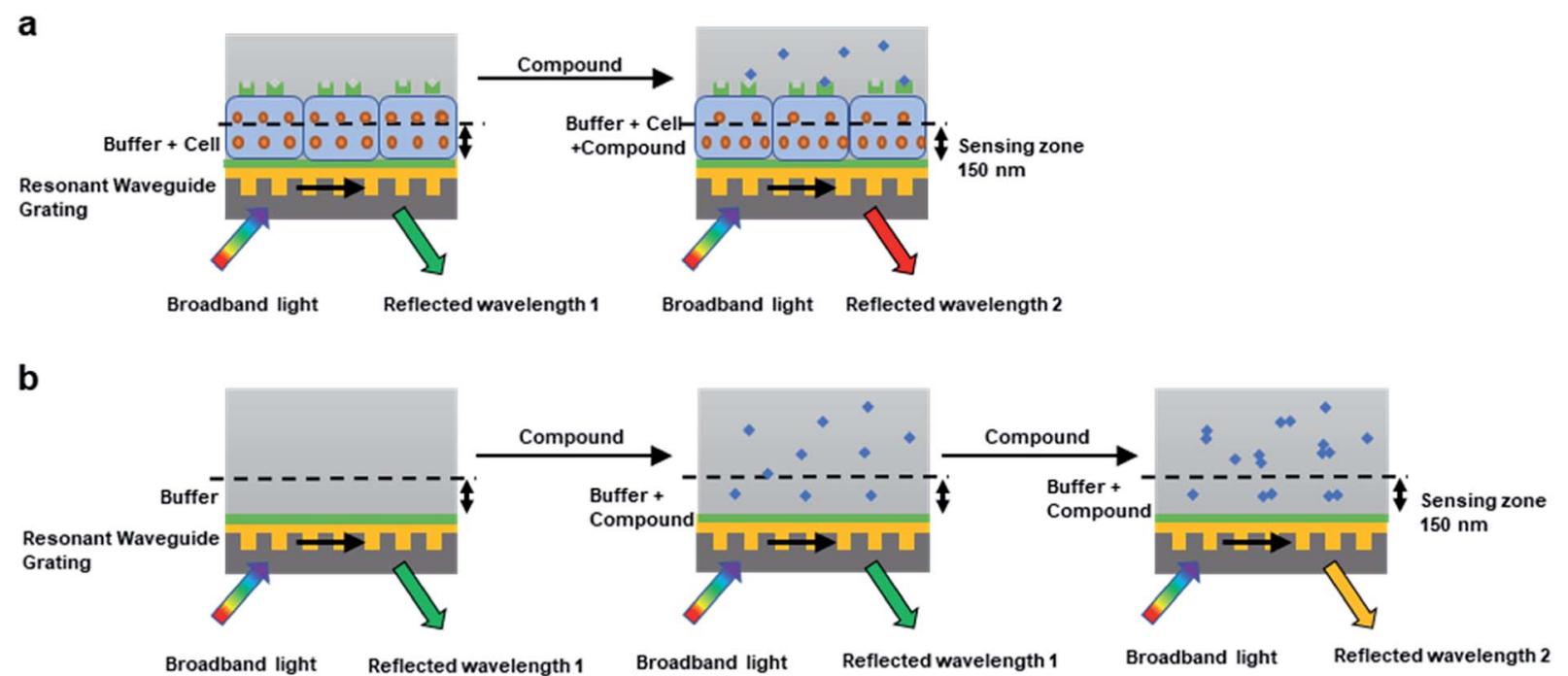

Fig. 1 Principles of cell-based DMR assay and cell-free aggregation assay. (a) Cell-based DMR assay for biological test. (b) Cell-free aggregation assay for aggregates detection. 

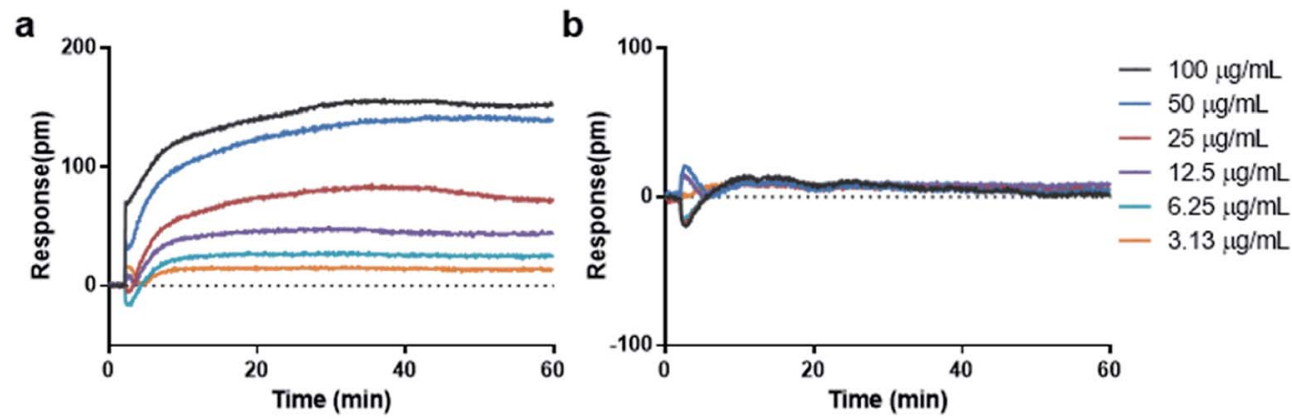

Fig. 2 Real time dose responses of representative aggregator and non-aggregator in aggregation assay. (a) Curcumin, aggregator. (b) Nicotinic acid, non-aggregator.

as a function of compound concentration is illustrated in Fig. 3 . The horizontal line represented non-aggregating state, and the oblique line represented aggregating state. The intersection of two straight lines was the critical aggregation concentration (CAC). Result showed that most of the compounds exhibited CAC values at micromolar scale (Table 1), which was nearly to previous reports. ${ }^{12,16}$

Overall, the cell-free aggregation assay implementing 384well microplate can examine compound aggregation and determine the CACs of small molecules in high throughput manner. Furthermore, the detection condition and process are exactly the same with the cell-environment and assay procedure, which can well mimic the external environment of cell surface.

\subsection{Characterization of aggregators' effect on cell-based DMR assays}

Four compounds, including procyanidin, salvianolic acid C, quercetin and curcumin, which represented the typical structures of the seven aggregation compounds, were used for the study. Three types of cell-based DMR assays, including DMR agonism assay, DMR desensitization assay and DMR antagonism assay, were adopted to characterize the aggregators' influence.

DMR agonism assay, which records the signal directly stimulated by the screening compound, was used to characterize the aggregators' direct effect on HT-29 cells. Results showed that procyanidins and salvianolic acid $\mathrm{C}$ triggered unusually large DMR responses in HT-29 cells, with the highest response of more than 1000 and 1500 pm, respectively, while at lower concentrations, they exhibited much lower responses (Fig. 4a). Quercetin triggered very low DMR response with less than $100 \mathrm{pm}$ at $100 \mu \mathrm{M}$, and curcumin barely triggered any cellular mass redistributions to its solubility limit (Fig. 4a). These results suggested that procyanidins and salvianolic acid C may non-specifically activated multiple ligands on cell surface and implied the promiscuity, while quercetin and curcumin did not.

After direct stimulation by the above four compounds, DMR desensitization assays were used to record receptor's response
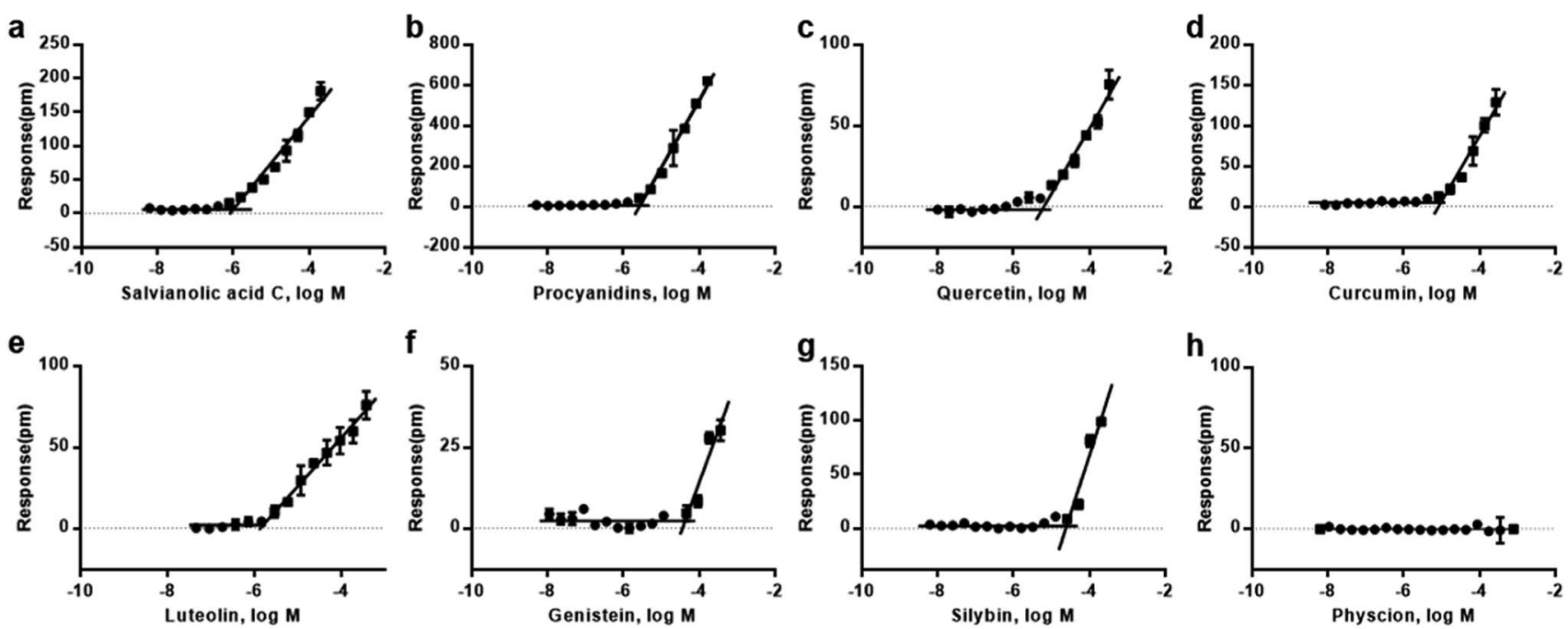

Fig. 3 Critical aggregation curves for the eight natural compounds. (a) Salvianolic acid C, (b) procyanidins, (c) quercetin, (d) curcumin, (e) luteolin, (f) genistein, (g) silybin, (h) physcion. The buffer employed for these studies was: $1 \times$ Hank's balanced salt solution (HBSS) buffer, 20 mM HEPES, $\mathrm{pH} 7.2$. 
Table 1 Critical aggregation concentrations for the eight natural compounds

\begin{tabular}{|c|c|c|c|}
\hline Compound & Structure & Detected CAC $(\mu \mathrm{M})$ & $\begin{array}{l}\text { Literature CAC detected } \\
\text { by DLS }(\mu \mathrm{M})\end{array}$ \\
\hline Salvianolic acid C & & 1.0 & - \\
\hline Procyanidins & & 2.8 & - \\
\hline Quercetin & & 5.7 & - \\
\hline Luteolin & & 1.6 & $<50$ (ref. 12) \\
\hline Genistein & & 42.8 & $<75$ (ref. 12) \\
\hline Silybin & & 25.2 & 0.47 (ref. 16) \\
\hline
\end{tabular}

upon its agonist stimulation. Muscarinic M3 receptor, which is endogenously expressed on HT-29 cells, ${ }^{43}$ was chosen for this assay. Since the above four compounds bear little physical or topological similarity with known ligands of M3, we expected the monomeric forms of the compounds to be relatively inert to the target. Thus, the activity would be an indication of promiscuity in itself. Results showed that both procyanidins and salvianolic acid $\mathrm{C}$ (at concentrations above $50 \mu \mathrm{M}$ ) inhibited the DMR response of acetylcholine against muscarinic M3 receptor (Fig. 4b). Quercetin, which exhibited normal response in the agonism assay, was also observed to false inhibited acetylcholine response at concentrations above $50 \mu \mathrm{M}$ (Fig. 4b). While the typical aggregator curcumin, did not inhibit acetylcholine response (Fig. 4b). These results indicated that aggregators may false inhibit membrane receptors. The $\mathrm{IC}_{50}$ values of the three promiscuous compounds were all greater than CAC value, which further suggested the aggregator-induced false activities (Table $\mathrm{S} 1 \dagger$ ). For procyanidins and salvianolic acid $\mathrm{C}$ with extremely high responses in the DMR agonism assay, the false inhibition could be derived from the aggregators' binding with M3 regions on cell surface. While for quercetin with low cell response in DMR agonism assay, this compound was more likely to interact with acetylcholine, and it was the experimental artifact that lead to the desensitization. The case of curcumin indicated that compound aggregation does not guarantee false inhibition of receptors.

The DMR antagonism assay records the response of screen compound on cells which is pre-blocked by receptor antagonist, therefore procyanidins and salvianolic acid $\mathrm{C}$ with high responses in agonism assay were chosen. The response of 100 $\mu \mathrm{M}$ procyanidins and salvianolic acid $\mathrm{C}$ were not dosedependently blocked by M3 antagonist atropine (Fig. 4c), or by the GPR35 antagonist ML145 (Fig. 4d). This result suggested that the DMR antagonism assay can help to identify the false results.

The above three cell-based DMR assays demonstrated that, aggregation does not guarantee false activity on cell-based receptor assays, but it is a worry sign. Colloidal aggregates may cause false activity in DMR desensitization assays, some of the false activities can be implied by the large DMR response in DMR agonism assays and be identified by DMR antagonism 
a

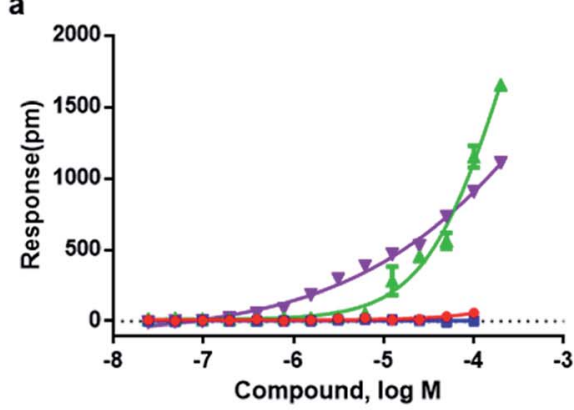

C

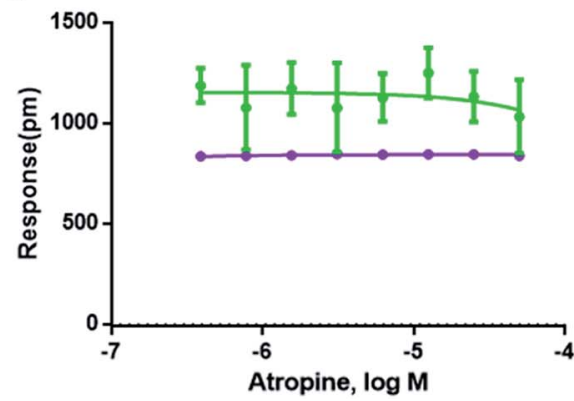

b

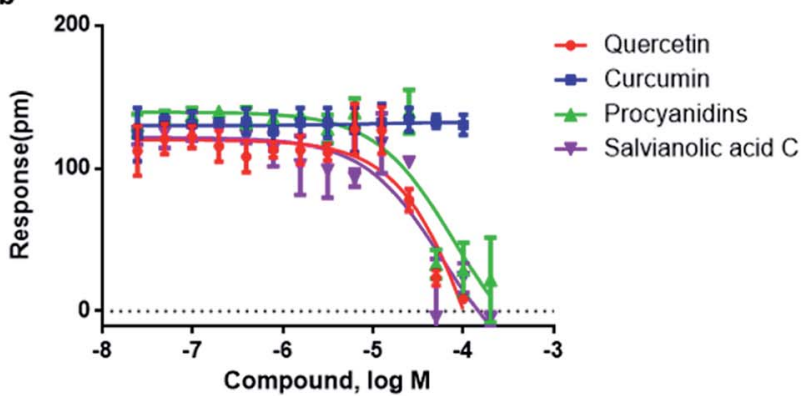

d

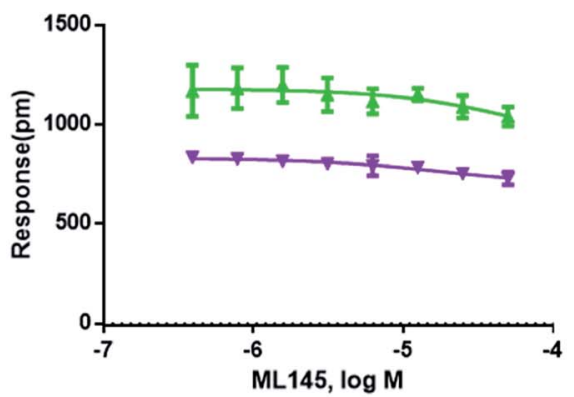

Fig. 4 DMR dose responses of the aggregators in HT-29 cells. (a) The DMR amplitudes of quercetin, curcumin, procyanidins and salvianolic acid $C$ as a function of their doses. (b) The DMR amplitudes of $16 \mu \mathrm{M}$ acetylcholine as a function of doses of quercetin, curcumin, procyanidins and salvianolic acid C. (c) The DMR amplitudes of $100 \mu$ M salvianolic acid C and procyanidins as a function of atropine. (d) The DMR amplitudes of 100 $\mu \mathrm{M}$ salvianolic acid $C$ and procyanidins as a function of ML145 doses.

assays. While for aggregators with low cell response, the identification could be more challenging.

Since the CAC was different for all the four compounds chosen, the relationship between CAC values and the DMR response was investigated (Fig. S1 $\dagger$ ). The responses of compounds at $100 \mu \mathrm{M}$ which clearly reflected the aggregation influence were selected. Result showed that DMR responses was somewhat correlated with CAC values: the lower CAC value, the higher DMR agonism response and the lower DMR desensitization response. These results suggested that the lower CAC value, the higher possibility of false activity. However, the relationship was not strictly proportional, for example, although quercetin exhibited larger CAC value than salvianolic acid $\mathrm{C}$ and procyanidins, it triggered lower response in the desensitization assay and exhibited false activity anyway. The possible explanation was that, although CAC value is an indication of the compounds' tendency in aggregation, not all aggregators lead to false activities; even for the aggregators that do result in false activities, they vary degrees. In addition, since the aggregators may interact with the activity results through different mechanisms, they may result in very different DMR responses.

\subsection{Detergent effect to colloidal aggregates' responses on cell-based DMR assays}

To further confirm the mechanism of the false results, we investigated the detergent effect on NP aggregates in three types of DMR assays. The detergent assay has been widely used for revealing the mechanism of aggregation induced false results. ${ }^{24,44,45}$ Tween- 80 with the concentration of $0.025 \%$ which exhibited tolerable disturbance on cell response was adopted as the working solution.

In the detergent-based DMR agonism assay, both procyanidins and salvianolic acid $\mathrm{C}$ showed decreased efficacy and potency (Fig. 5a and b). In the detergent-based DMR desensitization assay, GPR35 receptor with higher response and more robust signals was used to illustrate the effect. Results showed that both procyanidins and salvianolic acid C exhibited decreased potency on addition of tween-80, resulting in the increase of $\mathrm{IC}_{50}$ values from 14.13 to $65.64 \mu \mathrm{M}, 2.03$ to $8.65 \mu \mathrm{M}$, for procyanidins and salvianolic acid C, respectively (Fig. $5 \mathrm{c}$ and d). In the detergent-based DMR antagonism assay, the signals of salvianolic acid $\mathrm{C}$ and procyanidins were also declined significantly (Fig. 5e and f).

The above three cell-based detergent DMR assays further proved compound aggregation as the origin of false activities. At low concentration, soluble single molecule works on the real targets, while at high concentration, the colloidal aggregators bind other targets non-specifically, causing false results on agonism, desensitization and antagonism assays.

\subsection{Application of RWG based assays to eliminate false result in NPs}

Natural products is one of the promising sources for new drugs. ${ }^{46}$ However, the presence of aggregate compounds in NPs may impede their further development. Herein, Rhodiola rosea, a famous traditional herbal medicine, which is rich in polyphenols and flavonoids, ${ }^{47}$ was taken as an example to test the 
a

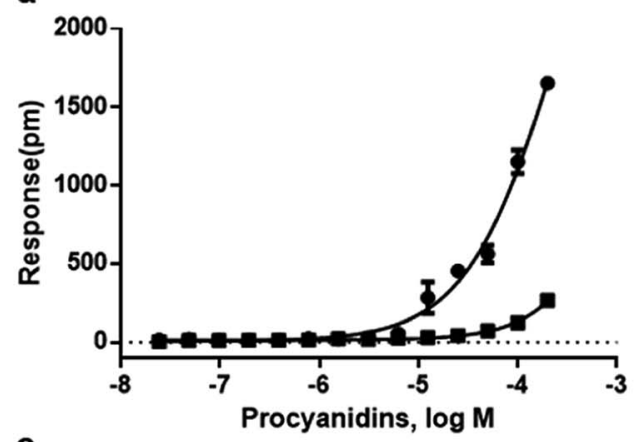

C

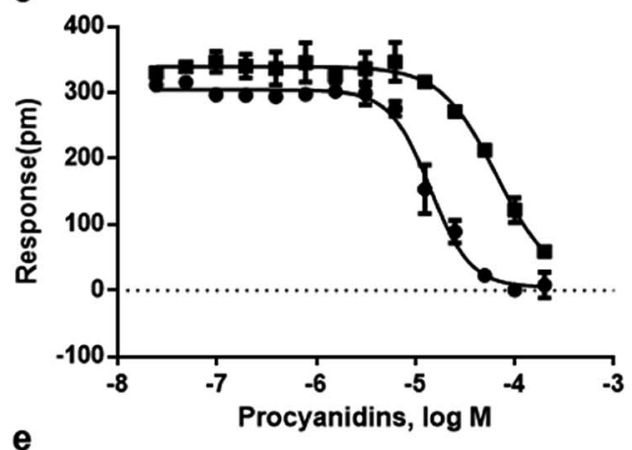

e

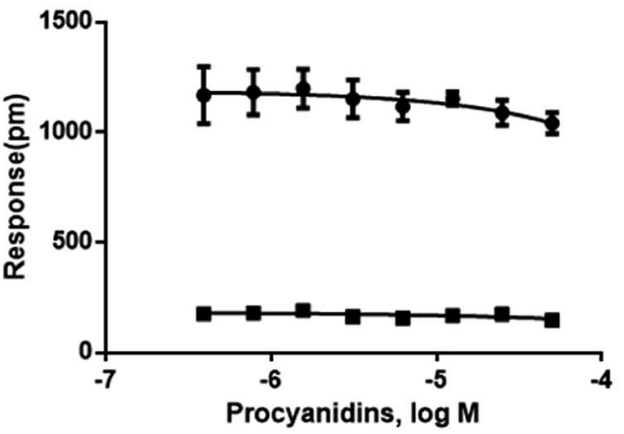

b

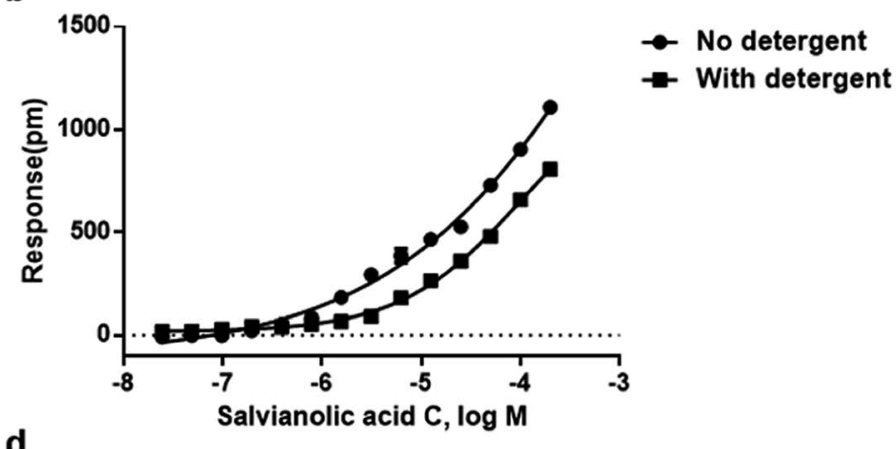

d
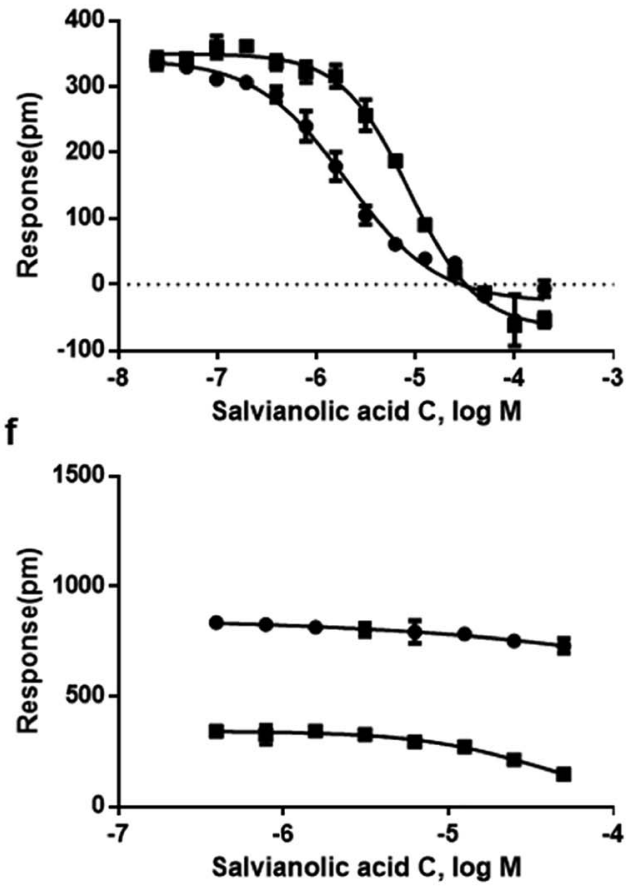

Fig. 5 Detergent effect on the DMR dose responses of the aggregators in HT-29 cells. (a) The DMR amplitudes as a function of procyanidins doses in the presence and absence of detergent. (b) The DMR amplitudes as a function of salvianolic acid C doses in the presence and absence of detergent. (c) The DMR amplitudes of $1 \mu \mathrm{M}$ zaprinast as a function of procyanidins doses in the presence and absence of detergent. (d) The DMR amplitudes of $1 \mu \mathrm{M}$ zaprinast as a function of salvianolic acid $C$ doses in the presence and absence of detergent. (e) The DMR amplitudes of 100 $\mu \mathrm{M}$ procyanidins as a function of ML145 doses in the presence and absence of detergent. (f) The DMR amplitudes of $100 \mu \mathrm{M}$ salvianolic acid C as a function of ML145 doses in the presence and absence of detergent.

feasibility of RWG based assays to eliminate the aggregates induced false active in complex mixtures.

We first used cell-free aggregation assay to test if the crude extract of Rhodiola rosea easily gets self-aggregate, result showed that the crude extract triggered extremely high response at the concentration of $50 \mu \mathrm{g} \mathrm{mL}^{-1}$, suggesting the formation of aggregates (Fig. 6a). After that, cell-based DMR assays were used to evaluate the promiscuity. DMR agonism assay with high response suggested the high possibility of aggregate induced promiscuity, and this was further proved by the detergent assay (Fig. 6b). We next performed DMR desensitization assays on several unrelated targets including M3, GPR35, neurotensin receptor (NTSR) and epidermal growth factor receptor (EGFR). The concentrations selected were 50, 25, $12.5 \mu \mathrm{g} \mathrm{mL}^{-1}$, which were routinely used for natural extracts screening. Results showed that all the receptors were dose-dependent desensitized at theses concentrations (Fig. 7a-d). In combination with DMR agonism assay and the corresponding detergent assay, we believed that the desensitization of these targets was promiscuous result rather than the multi-target effect arising from the multi-components in the extract.

To eliminate the promiscuous activities, the crude extract was next processed with a positive charged resin. We then examined the response of the treated extract on cell-free aggregation assay at the concentration of $50 \mu \mathrm{g} \mathrm{mL}{ }^{-1}$. Result showed that no significant response was observed (Fig. 6a). The HPLC analysis also proved the disappearance of the "hump" which was supposed to be polyphenols (Fig. 6c). These results suggested that the aggregators in the crude extract was successfully removed. A further examination of the treated 

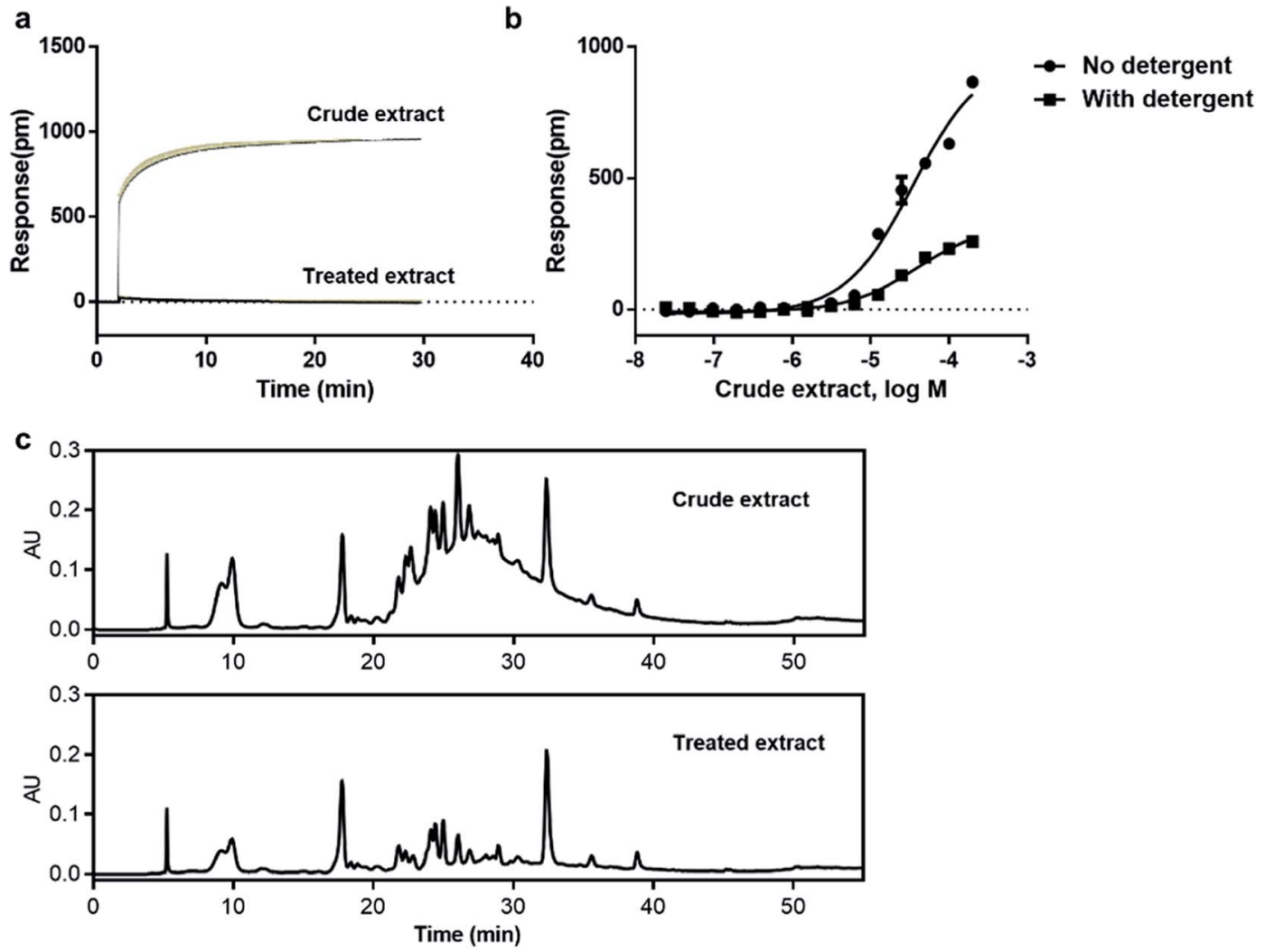

Fig. 6 Comparison between the crude extract and the treated extract. (a) Real time responses of $50 \mu \mathrm{g} \mathrm{mL} \mathrm{L}^{-1}$ of $\mathrm{crude} \mathrm{extract} \mathrm{and} 50 \mu \mathrm{g} \mathrm{mL}^{-1}$ of treated extract on cell-free aggregation assay. (b) The DMR amplitudes of crude extract as a function of their doses in the presence and absence of detergent. (c) HPLC/UV analysis of the crude extract and the treated extract at the UV wavelength of $270 \mathrm{~nm}$.

extract on DMR desensitization assays indicated that only GPR35 receptor was dose-dependently desensitized, while other three targets remained unaffected (Fig. 7e-h).
Taken together, RWG based assays can detect NP aggregators and make the activity results more reliable in the discovery of lead compounds, and therefore reduce the risk in bioassayguided drug discovery procedure.
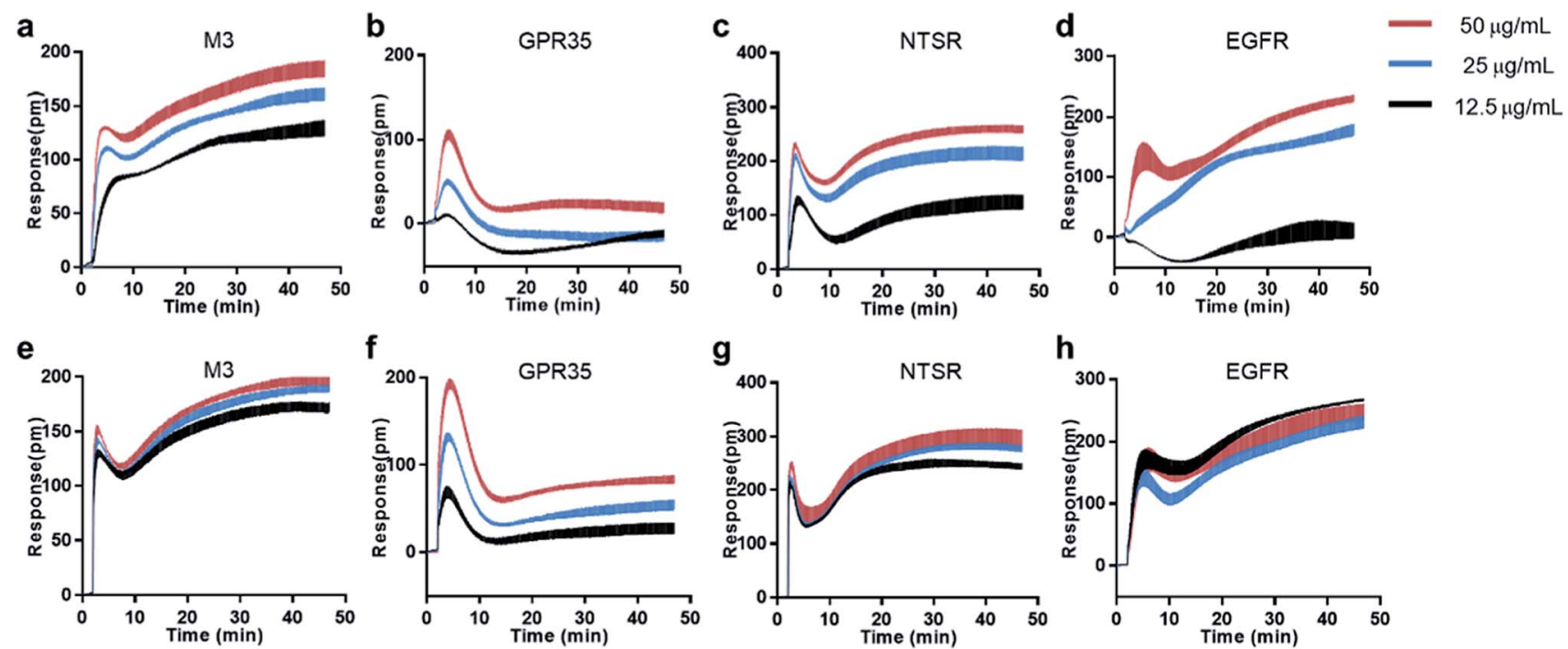

Fig. 7 Real time DMR responses of receptor agonists as a function of the dosed crude extract (up) and the dosed treated extract (down) in HT-29 cells. (a and e) $16 \mu \mathrm{M}$ acetylcholine, (b and f) $1 \mu \mathrm{M}$ zaprinast, (c and g) 2 nM neurotensin, ( $\mathrm{f}$ and h) $2 \mathrm{nM}$ EGF. 


\section{Conclusions}

In this work, a series of RWG based assays for colloidal aggregates detection and their promiscuity characterization were developed. Moreover, these assays were performed on a single platform, therefore the detecting and controls for aggregation could be readily conducted. Cell-free aggregation assay was first used to directly characterize eight well-known aggregate compounds and their critical aggregation concentrations. Then, cell-based DMR assays were performed to investigate the effect of natural compound aggregators on GPCRs. On addition of tween-80, the promiscuous compounds displayed decreased potency and efficacy. Finally, these assays were successfully applied to the detection of NP extract aggregators and made the activity result more reliable. This study highlights the great potential of RWG based assays in the detection of compound aggregators and helps to eliminate the false activities in drug discovery from natural products.

\section{Conflicts of interest}

The authors declare no competing financial interest.

\section{Acknowledgements}

This work was supported by the State Key Program of National Natural Science of China (U1508221), Project of National Science Foundation of China (81803706), Project of National Science Foundation of China (31670374), innovation program of science and research from DICP, CAS (DICP ZZBS201803) and the funding for the construction of DICP-CMC Innovation Institute of Medicine.

\section{References}

1 R. Macarron, M. N. Banks, D. Bojanic, D. J. Burns, D. A. Cirovic, T. Garyantes, D. V. Green, R. P. Hertzberg, W. P. Janzen and J. W. Paslay, Impact of high-throughput screening in biomedical research, Nat. Rev. Drug Discovery, 2011, 10, 188-195.

2 J. B. Baell, Screening-based translation of public research encounters painful problems, ACS Med. Chem. Lett., 2015, 6, 229-234.

3 R. F. Bruns and I. A. Watson, Rules for identifying potentially reactive or promiscuous compounds, J. Med. Chem., 2012, 55, 9763-9772.

4 S. Zhou, E. Chan, W. Duan, M. Huang and Y. Z. Chen, Drug bioactivation covalent binding to target proteins and toxicity relevance, Drug Metab. Rev., 2005, 37, 41-213.

5 A. B. Shapiro, G. K. Walkup and T. A. Keating, Correction for interference by test samples in high-throughput assays, $J$. Biomol. Screening, 2009, 14, 1008-1016.

6 A. Simeonov, A. Jadhav, C. J. Thomas, Y. Wang, R. Huang, N. T. Southall, P. Shinn, J. Smith, C. P. Austin and D. S. Auld, Fluorescence spectroscopic profiling of compound libraries, J. Med. Chem., 2008, 51, 2363-2371.
7 D. S. Auld, N. T. Southall, A. Jadhav, R. L. Johnson, D. J. Diller, A. Simeonov, C. P. Austin and J. Inglese, Characterization of chemical libraries for luciferase inhibitory activity, J. Med. Chem., 2008, 51, 2372-2386.

8 B. Y. Feng, A. Simeonov, A. Jadhav, K. Babaoglu, J. Inglese, B. K. Shoichet and C. P. Austin, A high-throughput screen for aggregation-based inhibition in a large compound library, J. Med. Chem., 2007, 50, 2385-2390.

9 N. Thorne, D. S. Auld and J. Inglese, Apparent activity in high-throughput screening: origins of compounddependent assay interference, Curr. Opin. Chem. Biol., 2010, 14, 315-324.

10 B. Y. Feng, A. Shelat, T. N. Doman, R. K. Guy and B. K. Shoichet, High-throughput assays for promiscuous inhibitors, Nat. Chem. Biol., 2005, 1, 146-148.

11 S. L. McGovern, B. T. Helfand, B. Feng and B. K. Shoichet, A specific mechanism of nonspecific inhibition, J. Med. Chem., 2003, 46, 4265-4272.

12 M. F. Sassano, A. K. Doak, B. L. Roth and B. K. Shoichet, Colloidal aggregation causes inhibition of $\mathrm{G}$ proteincoupled receptors, J. Med. Chem., 2013, 56, 2406-2414.

13 K. E. Coan, D. A. Maltby, A. L. Burlingame and B. K. Shoichet, Promiscuous aggregate-based inhibitors promote enzyme unfolding, J. Med. Chem., 2009, 52, 2067-2075.

14 K. E. Coan and B. K. Shoichet, Stoichiometry and physical chemistry of promiscuous aggregate-based inhibitors, $J$. Am. Chem. Soc., 2008, 130, 9606-9612.

15 S. C. Owen, A. K. Doak, P. Wassam, M. S. Shoichet and B. K. Shoichet, Colloidal aggregation affects the efficacy of anticancer drugs in cell culture, ACS Chem. Biol., 2012, 7, 1429-1435.

16 D. Duan, A. K. Doak, L. Nedyalkova and B. K. Shoichet, Colloidal aggregation and the in vitro activity of traditional Chinese medicines, ACS Chem. Biol., 2015, 10, 978-988.

17 R. Sink, S. Gobec, S. Pecar and A. Zega, False positives in the early stages of drug discovery, Curr. Med. Chem., 2010, 17, 4231-4255.

18 M. G. Weller, A unifying review of bioassay-guided fractionation, effect-directed analysis and related techniques, Sensors, 2012, 12, 9181-9209.

19 D. Camp, R. A. Davis, E. A. Evans-Illidge and R. J. Quinn, Guiding principles for natural product drug discovery, Future Med. Chem., 2012, 4, 1067-1084.

20 J. B. Baell, Feeling nature's PAINS: natural products, natural product drugs, and pan assay interference compounds (PAINS), J. Nat. Prod., 2016, 79, 616-628.

21 J. Bisson, J. B. McAlpine, J. B. Friesen, S.-N. Chen, J. Graham and G. F. Pauli, Can invalid bioactives undermine natural product-based drug discovery?, J. Med. Chem., 2015, 59, 1671-1690.

22 A. G. Atanasov, B. Waltenberger, E.-M. Pferschy-Wenzig, T. Linder, C. Wawrosch, P. Uhrin, V. Temml, L. Wang, S. Schwaiger and E. H. Heiss, Discovery and resupply of pharmacologically active plant-derived natural products: A review, Biotechnol. Adv., 2015, 33, 1582-1614. 
23 C. J. Henrich and J. A. Beutler, Matching the power of high throughput screening to the chemical diversity of natural products, Nat. Prod. Rep., 2013, 30, 1284-1298.

24 S. L. McGovern, E. Caselli, N. Grigorieff and B. K. Shoichet, A common mechanism underlying promiscuous inhibitors from virtual and high-throughput screening, J. Med. Chem., 2002, 45, 1712-1722.

25 J. Seidler, S. L. McGovern, T. N. Doman and B. K. Shoichet, Identification and prediction of promiscuous aggregating inhibitors among known drugs, J. Med. Chem., 2003, 46, 4477-4486.

26 C. Dalvit, D. Caronni, N. Mongelli, M. Veronesi and A. Vulpetti, NMR-based quality control approach for the identification of false positives and false negatives in high throughput screening, Curr. Drug Discovery Technol., 2006, 3, 115-124.

27 S. R. LaPlante, R. Carson, J. Gillard, N. Aubry, R. Coulombe, S. Bordeleau, P. Bonneau, M. Little, J. O'Meara and P. L. Beaulieu, Compound aggregation in drug discovery: implementing a practical NMR assay for medicinal chemists, J. Med. Chem., 2013, 56, 5142-5150.

28 S. R. LaPlante, N. Aubry, G. Bolger, P. Bonneau, R. Carson, R. Coulombe, C. Sturino and P. L. Beaulieu, Monitoring drug self-aggregation and potential for promiscuity in offtarget in vitro pharmacology screens by a practical NMR strategy, J. Med. Chem., 2013, 56, 7073-7083.

29 J. R. Huth, R. Mendoza, E. T. Olejniczak, R. W. Johnson, D. A. Cothron, Y. Liu, C. G. Lerner, D. Chen and P. J. Hajduk, ALARM NMR: a rapid and robust experimental method to detect reactive false positives in biochemical screens Huth, J. Am. Chem. Soc., 2005, 127, 217-224.

30 D. H. Randle, K. A. Krebs, H. J. Gitschier and T. M. Upton, Label-Free Detection of Compound Aggregation Using Corning® Epic® Technology, https://www.corning.com/ media/worldwide/cls/documents/snappshots_Label-Free_ Detection_of_Compound_Aggregation_using_Corning_ Epic_technology.pdf, 2011.

31 J. M. Blevitt, M. D. Hack, K. L. Herman, P. F. Jackson, P. J. Krawczuk, A. D. Lebsack, A. X. Liu, T. Mirzadegan, M. I. Nelen and A. N. Patrick, Structural basis of smallmolecule aggregate induced inhibition of a protein-protein interaction, J. Med. Chem., 2017, 60, 3511-3517.

32 Y. Fang, Label-free cell-based assays with optical biosensors in drug discovery, Assay Drug Dev. Technol., 2006, 4, 583-595.

33 A. M. Ferrie, Q. Wu and Y. Fang, Resonant waveguide grating imager for live cell sensing, Appl. Phys. Lett., 2010, 97, 223704.
34 Y. Fang, A. M. Ferrie, N. H. Fontaine and P. K. Yuen, Characteristics of dynamic mass redistribution of epidermal growth factor receptor signaling in living cells measured with label-free optical biosensors, Anal. Chem., 2005, 77, 5720-5725.

35 Y. Fang, A. M. Ferrie, N. H. Fontaine, J. Mauro and J. Balakrishnan, Resonant waveguide grating biosensor for living cell sensing, Biophys. J., 2006, 91, 1925-1940.

36 N. Orgovan, B. Kovacs, E. Farkas, B. 1. SzabÃ, N. Zaytseva, Y. Fang and R. Horvath, Bulk and surface sensitivity of a resonant waveguide grating imager, Appl. Phys. Lett., 2014, 104, 083506.

37 Y. Fang, G. Li and A. M. Ferrie, Non-invasive optical biosensor for assaying endogenous $\mathrm{G}$ protein-coupled receptors in adherent cells, J. Pharmacol. Toxicol. Methods, 2007, 55, 314-322.

38 Y. Fang, A. G. Frutos and R. Verklereen, Label-free cell-based assays for GPCR screening, Comb. Chem. High Throughput Screening, 2008, 11, 357-369.

39 R. Schröder, J. Schmidt, S. BlÃttermann, L. Peters, N. Janssen, M. Grundmann, W. Seemann, D. Kaufel, N. Merten and C. Drewke, Applying label-free dynamic mass redistribution technology to frame signaling of $\mathrm{G}$ protein-coupled receptors noninvasively in living cells, Nat. Protoc., 2011, 6, 1748-1760.

40 Y. Fang, Non-invasive optical biosensor for probing cell signaling, Sensors, 2007, 7, 2316-2329.

41 J. Baell and M. A. Walters, Chemistry: Chemical con artists foil drug discovery, Nature, 2014, 513, 481-483.

42 L. Pohjala and P. i. Tammela, Aggregating behavior of phenolic compounds-a source of false bioassay results?, Molecules, 2012, 17, 10774-10790.

43 H. Deng, C. Wang, M. Su and Y. Fang, Probing biochemical mechanisms of action of muscarinic M3 receptor antagonists with label-free whole cell assays, Anal. Chem., 2012, 84, 8232-8239.

44 A. J. Ryan, N. M. Gray, P. N. Lowe and C.-w. Chung, Effect of detergent on "promiscuous" inhibitors, J. Med. Chem., 2003, 46, 3448-3451.

45 B. Y. Feng and B. K. Shoichet, A detergent-based assay for the detection of promiscuous inhibitors, Nat. Protoc., 2006, 1, 550-553.

46 A. L. Harvey, R. Edrada-Ebel and R. J. Quinn, The reemergence of natural products for drug discovery in the genomics era, Nat. Rev. Drug Discovery, 2015, 14, 111-129.

47 A. Panossian, G. Wikman and J. Sarris, Rosenroot (Rhodiola rosea): traditional use, chemical composition, pharmacology and clinical efficacy, Phytomedicine, 2010, 17, 481-493. 